\title{
NORM INEQUALITIES RELATING ONE-SIDED SINGULAR INTEGRALS AND THE ONE-SIDED MAXIMAL FUNCTION
}

\author{
M. S. RIVEROS and A. DE LA TORRE
}

(Received 9 December 1998; revised 17 April 2000)

Communicated by P. G. Dodds

\begin{abstract}
In this paper we prove that if a weight $w$ satisfies the $C_{q}^{+}$condition, then the $L^{p}(w)$ norm of a one-sided singular integral is bounded by the $L^{p}(w)$ norm of the one-sided Hardy-Littlewood maximal function, for $1<p<q<\infty$.

2000 Mathematics subject classification: primary 42B25, 42 A50.

Keywords and phrases: One-sided weights, one-sided singular integrals.
\end{abstract}

\section{Introduction}

One-sided singular integrals were defined by Aimar, Forzani and Martín-Reyes in [AFM] as singular integrals $T^{+} f$ whose kernel has support on $(-\infty, 0)$. In the same paper they proved that a weight $w$ satisfies $\int\left|T^{+} f\right|^{p} w \leq C \int|f|^{p} w$, for all $f \in L^{p}(w)$ if, the weight satisfies the one-sided $A_{p}^{+}$condition, introduced by Sawyer [S1], that characterizes the boundedness of the one-sided Hardy-Littlewood maximal operator $M^{+} f(x)=\sup _{h>0} h^{-1} \int_{x}^{x+h}|f|$.

A crucial step in the proof, is the fact that if $w \in A_{\infty}^{+}$, then

$$
\int\left|T^{+} f\right|^{r} w \leq C \int\left[M^{+} f\right]^{r} w
$$

for any $1<r$. We recall definitions of the $A_{p}^{+}$classes: $w \in A_{p}^{+}, 1<p$ if there exists a constant $C$ such that for all $a<b<c$

Research supported by D.G.E.S (PB97-1097), Junta de Andalucía and Universidad Nacional de Córdoba.

(C) 2000 Australian Mathematical Society 0263-6115/2000\$A2.00+0.00 
$\left(A_{p}^{+}\right)$

$$
\int_{a}^{b} w\left(\int_{b}^{c} w^{1-p^{\prime}}\right)^{p-1} \leq C(c-a)^{p}
$$

where $p+p^{\prime}=p p^{\prime}$. A weight $w$ is in $A_{\infty}^{+}$if there exist positive constants $C$ and $\epsilon$ such that for any $a<b<c$ and any measurable set $E \subset(a, b)$,

$$
\frac{\int_{E} w}{\int_{a}^{c} w} \leq C\left(\frac{|E|}{c-b}\right)^{\epsilon} .
$$

These definitions and many properties of $A_{p}^{+}$and $A_{\infty}^{+}$can be found in [MPT]. A natural question arises. Can we find conditions weaker than $A_{\infty}^{+}$that are sufficient for (1.1)? In [S2] Sawyer considered the following condition, introduced first by Muckenhoupt in $[\mathrm{Mu}]$.

There exists two positive constants $C$ and $\epsilon$ such that for every interval $I \in \mathbb{R}$ and every measurable subset $E \subset I$ we have

$$
\int_{E} w \leq C\left(\frac{|E|}{|I|}\right)^{\epsilon} \int\left[M \chi_{l}\right]^{p} w<\infty,
$$

where $M$ is the Hardy-Littlewood maximal operator. Sawyer proved that for a standard singular integral $T f, C_{q}$ is suficient for

$$
\int|T f|^{p} w \leq C \int[M f]^{p} w
$$

provided $q>p$. He does not require $\int\left[M_{\chi_{I}}\right]^{p} w<\infty$. Observe that if $\int\left[M_{\chi_{I}}\right]^{q} w=\infty$ for some 1 , then $\int\left[M \chi_{J}\right]^{q} w=\infty$ for every interval $J$. Then for every $f \geq 0$ and $p \leq q$ we have that $\int[M f]^{p} w=\infty$. In this paper we introduce a one-sided version of this condition $C_{p}^{+}$, and prove that if $q>p$, then

$$
\int\left|T^{+} f\right|^{p} w \leq C \int\left[M^{+} f\right]^{p} w .
$$

The definition of $C_{p}^{+}$is as follows.

DEFINITION. A weight $w$ satisfies $C_{p}^{+}$if there exist $\epsilon>0$ and $C>0$, so that for any $a<b<c$, with $c-b<b-a$, and any measurable set $E \subseteq(a, b)$, the following holds

$$
\int_{E} w \leq C\left(\frac{|E|}{(c-b)}\right)^{\epsilon} \int_{\mathbb{R}}\left[M^{+} \chi_{(a, c)}\right]^{p} w<\infty
$$


Observe that if $w \in A_{\infty}^{+}$then $w \in \bigcap_{p>1} C_{p}^{+}$. We give examples of weights that satisfy $C_{p}^{+}$condition for all $p>1$ but they do not satisfy $A_{\infty}^{+}$condition.

The class of one-sided singular integrals is a subclass of the standard singular integrals and our theorem says that for this subclass we can obtain a more precise result. On one hand, we obtain a smaller right hand side, with $M^{+} f$ instead of $M f$. On the other hand, the condition $C_{p}^{+}$is different from $C_{p}$. These facts make the proof more complicated than in the standard case although it follows the same lines as the paper by Sawyer.

Now we recall the definition of one-sided singular integrals studied in [AFM]. We say that a function $k$ in $L_{\text {loc }}^{1}(\mathbb{R}-\{0\})$ is a Calderón-Zygmund kernel if the following properties are satisfied:

(a) There exists a finite constant $B_{1}$ such that

$$
\left|\int_{\epsilon<|x|<N} k(x) d x\right| \leq B_{1}
$$

for all $\epsilon$ and all $N$ with $0<\epsilon<N$. Furthermore $\lim _{\epsilon \rightarrow 0^{+}} \int_{\epsilon<|x|<N} k(x) d x$ exists.

(b) There exists a finite constant $B_{2}$ such that

$$
|k(x)| \leq B_{2} /|x|
$$

for all $x \neq 0$.

(c) There exists a finite constant $B_{3}$ such that

$$
|k(x-y)-k(x)| \leq B_{3}|y||x|^{-2}
$$

for all $x$ and $y$ with $|x|>2|y|>0$.

A one-sided singular integral is

$$
T^{+} f(x)=\lim _{\epsilon \rightarrow 0} \int_{x+\epsilon}^{\infty} k(x-y) f(y) d y,
$$

where $k$ is a Calderon-Zygmund kernel, with support in $\mathbb{R}^{-}$. We also define

$$
T^{*+} f(x)=\sup _{\epsilon>0}\left|\int_{x+\epsilon}^{\infty} k(x-y) f(y) d y,\right|
$$

Examples of such kernels are given in [AFM].

We end this section with some notation. A weight $w$ is a non-negative, locally integrable function. If $E$ is a measurable set, $w(E)$ denotes the integral of $w$ over $E$. Throughout the paper the letter $C$ represents a positive constant that may change from time to time. 


\section{Statement and proof of the result}

THEOREM 1. Let $T^{+} f$ be a one-sided singular integral, $1<p<q<\infty$ and assume that $w$ satisfies $C_{q}^{+}$, then

$$
\int_{\mathbb{R}}\left|T^{+} f\right|^{p} w \leq C \int_{\mathbb{R}}\left[M^{+} f\right]^{p} w
$$

for all $f$ such that the right hand side is finite.

REMARK. If $w(x)=e^{x}$ then $w \in A_{1}^{+} \subset A_{\infty}^{+} \subset C_{p}^{+}, p>1$. But $\int\left[M \chi_{l}\right]^{p} w=\infty$, and therefore $w \notin C_{p}, p>1$.

The proof is based on a series of lemmas that we now state and prove.

LEMMA 1. Let us assume that $w$ satisfies $C_{q}^{+}, 1<q<\infty$, then for any $\delta>0$ there exists $C(\delta)$ such that for any disjoint family of intervals $\left\{J_{j}\right\}$ contained in $I=(a, b)$ we have

$$
\int_{l} \sum_{j}\left[M^{+} \chi_{J_{j}}\right]^{q} w \leq C(\delta) w(I)+\delta \int_{\mathbf{R}}\left[M^{+} \chi_{l}\right]^{q} w
$$

and

$$
\int_{\mathbf{R}} \sum_{j}\left[M^{+} \chi_{j_{j}}\right]^{q} w \leq C \int_{\mathbf{R}}\left[M^{+} \chi_{l}\right]^{q} w
$$

PROOF. First, we claim that (i) implies (ii). Indeed,

$$
\begin{aligned}
\int_{\mathbb{R}}\left(\sum_{j}\left[M^{+} \chi_{J_{j}}\right]^{q}\right) w & =\int_{I}\left(\sum_{j}\left[M^{+} \chi_{J_{j}}\right]^{q}\right) w+\int_{(-\infty, a)}\left(\sum_{j}\left[M^{+} \chi_{J_{j}}\right]^{q}\right) w \\
& \leq C(\delta) w(I)+\delta \int_{\mathbb{R}}\left[M^{+} \chi_{I}\right]^{q} w+\int_{(-\infty, a)} \frac{\sum_{j}\left|J_{j}\right|^{q}}{(b-x)^{q}} w \\
& \leq C(\delta) w(I)+\delta \int_{\mathbb{R}}\left[M^{+} \chi_{I}\right]^{q} w+\int_{(-\infty, a)} \frac{|I|^{q}}{(b-x)^{q}} w \\
& \leq C(\delta) w(I)+(\delta+1) \int_{\mathbb{R}}\left[M^{+} \chi_{I}\right]^{q} w \\
& \leq 2 C(\delta) \int_{\mathbb{R}}\left[M^{+} \chi_{I}\right]^{q} w+(\delta+1) \int_{\mathbb{R}}\left[M^{+} \chi_{I}\right]^{q} w .
\end{aligned}
$$

To prove (i) we use the fact that there exists $\alpha>0$ such that for every $\lambda>0$ we have

$$
\left|E_{\lambda}\right|=\left|\left\{x: \sum_{j}\left[M^{+} \chi_{J_{j}}\right]^{q}(x)>\lambda\right\}\right| \leq C e^{-\alpha \lambda}|I|
$$


(for details see [FeSt]). We define a sequence of points as follows: $x_{0}=a$ and for $i \in \mathbb{N}, x_{i}-x_{i-1}=b-x_{i}$ and consider the sets $E_{\lambda}^{i}=E_{\lambda} \cap\left(x_{i}, x_{i+1}\right)$. For $x \in\left(x_{i}, x_{i+1}\right)$ we may assume that $J_{j}$ in $\sum_{j}\left|M^{+} \chi_{J_{j}}\right|^{q}(x)$ are all contained in $\left(x_{i}, b\right)$. It follows from (2.1) that

$$
\left|E_{\lambda}^{i}\right| \leq C e^{-\alpha \lambda}\left(b-x_{i}\right)=C e^{-\alpha \lambda}\left(x_{i+2}-x_{i+1}\right) .
$$

If we now use condition $C_{q}^{+}$for the set $E_{\lambda}^{i}$ and the points $x_{i}, x_{i+1}, x_{i+2}$ we get

$$
w\left(E_{\lambda}^{i}\right) \leq C e^{-\alpha \lambda \epsilon} \int\left[M^{+} \chi_{\left(x_{i}, x_{i+2}\right)}\right]^{q} w .
$$

It is easy to see that $\sum_{i>1} M^{+} \chi_{\left(x_{i}, x_{i+2}\right)} \leq C M^{+} \chi_{I}$ and adding up we get

$$
w\left(E_{\lambda} \cap I\right) \leq C e^{-\alpha \lambda \epsilon} \int\left[M^{+} \chi_{I}\right]^{q} w .
$$

Therefore,

$$
\begin{aligned}
\int_{I} \sum_{j}\left[M^{+} \chi_{J_{j}}\right]^{q} w & =\int_{0}^{\lambda_{0}} \int_{E_{\lambda} \cap I} w d \lambda+\int_{\lambda_{0}}^{\infty} \int_{E_{\lambda} \cap I} w d \lambda \\
& \leq \lambda_{0} w(I)+\int_{\lambda_{0}}^{\infty} w\left(E_{\lambda} \cap I\right) d \lambda \\
& \leq \lambda_{0} w(I)+C \int_{\lambda_{0}}^{\infty} e^{-\alpha \lambda \epsilon} d \lambda \int\left[M^{+} \chi_{I}\right]^{q} w \\
& \leq C(\delta) w(I)+\delta \int\left[M^{+} \chi_{I}\right]^{q} w
\end{aligned}
$$

if we choose $\lambda_{0}$ big enough.

For the next lemma we need to define a new operator, $M_{p, q}^{+}$. Let $f$ be a nonnegative measurable function. Let us consider

$$
\Omega_{k}=\left\{x: f(x)>2^{k}\right\}=\bigcup_{i} I_{i}^{k},
$$

where $I_{i}^{k}$ are the connected components of $\Omega_{k}$. Then

$$
\left[M_{p, q}^{+} f(x)\right]^{p}=\sum_{k, i} 2^{p k}\left[M^{+} \chi_{I_{i}^{k}}(x)\right]^{q}
$$

LEMMA 2. Let $1<p<q<\infty, w \in C_{q}^{+}$, and $f$ non-negative, bounded and of compact support. Then

$$
\int\left[M_{p, q}^{+}\left(M^{+} f\right)\right] w \leq C \int\left[M^{+} f\right]^{p} w .
$$


ProOF. Let $\Omega_{k}=\left\{x: M^{+} f(x)>2^{k}\right\}=\bigcup_{j} I_{j}^{k}$, where $I_{j}^{k}$ are the connected components of $\Omega_{k}$. Let $N \geq 1$, note that $\Omega_{k} \subseteq \Omega_{k-N}$ for all $k$. Given a connected component of $\Omega_{k-N}, I_{i}^{k-N}$ we estimate $\left|\Omega_{k} \cap I_{i}^{k-N}\right|$. First, we put $f=g+h$ with $g=f \chi_{I_{i}^{k-N}}$. Observe that if $x \in I_{i}^{k-N}=(a, b)$, then $M^{+} h(x) \leq M^{+} f(b) \leq 2^{k-N}$. So if $x \in \Omega_{k} \cap I_{i}^{k-N}$, then

$$
M^{+} g(x) \geq M^{+} f-M^{+} h \geq 2^{k}-2^{k-N} \geq \frac{1}{2} 2^{k} .
$$

Now using the fact that the operator $M^{+}$is of weak type $(1,1)$ with respect to Lebesgue measure we get

$$
\begin{aligned}
\left|\Omega_{k} \cap I_{i}^{k-N}\right| & \leq\left|\left\{x: M^{+} g(x) \geq \frac{1}{2} 2^{k}\right\}\right| \leq C 2^{-k} \int g \\
& =C 2^{-k} \int_{I_{i}^{k-N}} f \leq C 2^{-k}\left|I_{i}^{k-N}\right| M^{+} f(a) \leq C 2^{-N}\left|I_{i}^{k-N}\right|
\end{aligned}
$$

Let $S(k)=2^{k p} \sum_{j} \int\left[M^{+} \chi_{l_{j}^{k}}\right]^{q} w$ and $S(k, N, i)=2^{k p} \sum_{j: l_{j}^{k} \leq l_{i}^{k-N}} \int\left[M^{+} \chi_{l_{j}^{k}}\right]^{q} w$. Then

$$
S(k, N, i)=2^{k p} \sum_{j: I_{j}^{k} \subseteq I_{i}^{k-N}} \int_{I_{i}^{k-N}}\left[M^{+} \chi I_{j}^{k}\right]^{q} w+2^{k p} \sum_{j: I_{j}^{k} \subseteq I_{i}^{k-N}} \int_{\left(I_{i}^{k-N}\right) c}\left[M^{+} \chi I_{j}^{k}\right]^{q} w=\mathrm{I}+\mathrm{II} .
$$

By Lemma 1

$$
\mathrm{I} \leq C(\delta) 2^{k p} w\left(I_{i}^{k-N}\right)+\delta 2^{k p} \int\left[M^{+} \chi_{i_{i}^{k-N}}\right]^{q} w,
$$

where $\delta>0$ is chosen later. Now, by (2.2)

$$
\begin{aligned}
\mathrm{II} & \leq C 2^{k p} \int_{-\infty}^{a} \frac{\sum\left|I_{j}^{k}\right|^{q}}{(b-x)^{q}} w \leq C 2^{k p} \int_{-\infty}^{a} \frac{\left(C 2^{-N}\left|I_{i}^{k-N}\right|\right)^{q}}{(b-x)^{q}} w \\
& \leq C 2^{N(p-q)} 2^{p(k-N)} \int\left[M^{+} \chi_{l_{i}^{k-N}}\right]^{q} w .
\end{aligned}
$$

So we get

$$
S(k)=\sum_{i} S(k, N, i) \leq C(\delta) 2^{k p} \sum_{i} w\left(l_{i}^{k-N}\right)+\left[\delta 2^{N p}+C 2^{N(p-q)}\right] S(k-N) .
$$

As $p<q$, we can choose $\delta$ small and $N$ big enough such that

Now

$$
S(k) \leq C(\delta) 2^{k p} w\left(\Omega_{k-N}\right)+\frac{1}{2} S(k-N) .
$$

$$
S_{M}=\sum_{k \leq M} S(k) \leq \frac{1}{2} S_{M}+C \int\left[M^{+} f\right]^{p} w
$$


for all $M$. If we prove that under the assumptions on $f$, we have $S_{M}<\infty$, we are finished. Let us suppose that supp $f \subset I=(a, b)$. There exists $L$ such that $2^{L}<1 /(b-a) \int_{a}^{b} f \leq 2^{L+1}$.

If $k \geq L+1$, then $\Omega_{k} \subset I^{-} \cup I$, where $I^{-}=(2 a-b, a)$. Indeed, if $x<2 a-b$, then

$$
M^{+} f(x)=\sup _{h>a-x>b-a} \frac{1}{h} \int_{x}^{x+h} \leq \frac{1}{b-a} \int_{a}^{b} f \leq 2^{L+1}
$$

If $l_{j}^{k}$ are the connected components of $\Omega_{k}$, using Lemma 1 and since $q>p$, we have

$$
\sum_{k=L+1}^{M} \sum_{j} 2^{k p} \int\left[M^{+} \chi_{I_{j}^{k}}\right]^{q} w \leq \sum_{k=L+1}^{M} 2^{k p} \int\left[M^{+} \chi_{I-\cup I}\right]^{q} w \leq C \int\left[M^{+} \chi_{I}\right]^{p} w<\infty .
$$

If $k \leq L$ we can show again that $\Omega_{k} \subset 2^{L-k+2}\left(I^{-}\right) \cup I$, where $2^{n}\left(I^{-}\right)=\left(c_{n}, a\right)$, with $\left(a-c_{n}\right)=2^{n}(b-a)$. Then by Lemma 1 we have

$$
\sum_{k \leq L} \sum_{j} 2^{k p} \int\left[M^{+} \chi_{I_{j}^{k}}\right]^{q} w \leq C \sum_{k \leq L} 2^{k p} \int\left[M^{+} \chi_{2^{L-k+2}\left(I^{-}\right) \cup I}\right]^{q} w .
$$

Now its easy to see, using $p<q$, that

$$
\sum_{k \leq L} 2^{k p}\left[M^{+} \chi_{2^{L-k+2}\left(I^{-}\right) \cup I}(x)\right]^{q} \leq C 2^{L p}\left[M^{+} \chi_{I}(x)\right]^{p}<\infty .
$$

LEMMA 3. Let $1<p<q<\infty, w \in C_{q}^{+}$and let $f$ be a non-negative bounded function with compact support. Then

$$
\int\left[M_{p, q}^{+}\left(T^{*+} f\right)\right]^{p} w \leq C\left[\int\left[T^{*+} f\right]^{p} w+\int\left[M^{+} f\right]^{p} w\right] .
$$

PROOF. Let $\Omega_{k}=\left\{x: T^{*+} f(x)>2^{k}\right\}=\bigcup_{j} I_{j}^{k}$, where $I_{j}^{k}$ are the connected components of $\Omega_{k}$. Observe that in the proof of the 'good lambda inequality' in [AFM, Lemma 2.7], what they really show is

(2.3) $\left|\left\{x \in I_{i}^{k-N}: T^{*+} f(x)>2^{k}\right\}\right| \leq C 2^{-N}\left|I_{i}^{k-N}\right| \quad$ if $I_{i}^{k-N} \nsubseteq\left\{x: M^{+} f(x)>2^{k-N}\right\}$.

Let $O_{k}=\left\{x: M^{+} f(x)>2^{k}\right\}=\bigcup_{j} J_{j}^{k}$, where $J_{j}^{k}$ are the connected components of $O_{k}$. For each $l_{i}^{k-N}$ we have two cases

(i) $I_{i}^{k-N} \subseteq O_{k-N}$,

(ii) $I_{i}^{k-N} \nsubseteq O_{k-N}$. 
Case (1). There exists $l_{i}$ such that $l_{i}^{k-N} \subseteq J_{l_{i}}^{k-N}$.

Case (2). (2.3) implies

$$
\sum_{j: I_{j}^{k} \leq I_{i}^{k-N}}\left|I_{j}^{k}\right|=\left|\left\{x \in I_{i}^{k-N}: T^{*+} f(x)>2^{k}\right\}\right| \leq C 2^{-N}\left|I_{i}^{k-N}\right| .
$$

Let $S(k)=2^{k p} \sum_{j} \int\left[M^{+} \chi_{l_{j}^{k}}\right]^{q} w$ and $S(k, N, i)=2^{k p} \sum_{j: I_{j}^{k} \subseteq I_{i}^{k-N}} \int\left[M^{+} \chi_{l_{j}^{k}}\right]^{q} w$. Then

$$
S(k, N, i)=2^{k p} \sum_{j \cdot I_{j}^{k} \subseteq I_{i}^{k-N}} \int_{I_{i}^{k-N}}\left[M^{+} \chi_{I_{i}^{k}}\right]^{q} w+2^{k p} \sum_{j: I_{j}^{k} \subseteq I_{i}^{k-N}} \int_{\left(I_{i}^{k-N}, c\right.}\left[M^{+} \chi_{I_{j}^{k}}\right]^{q} w=\mathrm{I}+\mathrm{II} .
$$

By Lemma 1 we have that

$$
\mathrm{I} \leq C(\delta) 2^{k p} w\left(I_{i}^{k-N}\right)+\delta 2^{k p} \int\left[M^{+} \chi_{i_{i}^{k-N}}\right]^{q} w,
$$

where $\delta>0$. We denote $\left(a_{i}^{k-N}, b_{i}^{k-N}\right)=l_{i}^{k-N}$, then by (2.4) we obtain

$$
\begin{aligned}
\mathrm{II} & \leq C 2^{k p} \int_{-\infty}^{a_{i}^{k-N}} \frac{\sum_{j: I_{j}^{k} \subseteq I_{i}^{k-N}}\left|I_{j}^{k}\right|^{q}}{\left(b_{i}^{k-N}-x\right)^{q}} w \leq C 2^{k p} \int_{-\infty}^{a_{i}^{k-N}} \frac{\left(C 2^{-N}\left|I_{i}^{k-N}\right|\right)^{q}}{\left(b_{i}^{k-N}-x\right)^{q}} w \\
& \leq C 2^{k p-N q} \int\left[M^{+} \chi I_{i}^{k-N}\right]^{q} w .
\end{aligned}
$$

Adding I and II we get

$$
S(k, N, i) \leq C(\delta) 2^{k p} w\left(I_{i}^{k-N}\right)+\left(\delta+C 2^{-N q}\right) 2^{k p} \int\left[M^{+} \chi_{i_{i}^{k-N}}\right]^{q} w .
$$

Then

$$
S(k)=\sum_{\substack{i: I_{i}^{k-N} \\ \text { is in case (1) }}} S(k, N, i)+\sum_{\substack{i: I_{i}^{k-N} \\ \text { is in case (2) }}} S(k, N, i)=\mathrm{III}+\mathrm{IV} .
$$

For III we observe that $I_{j}^{k}$ is contained in exatly one $J_{l}^{k-N}$ and by Lemma 1 we have

$$
\begin{aligned}
\mathrm{III} & =\sum_{i: I_{i}^{k-N} \subseteq J_{t_{i}^{k-N}}} S(k, N, i)=\sum_{i: I_{i}^{k-N} \subseteq J_{t_{i}^{k}}-N} \sum_{j: I_{j}^{k} \subseteq I_{i}^{k-N}} 2^{k p} \int\left[M^{+} \chi_{l_{j}^{k}}\right]^{q} w \\
& \leq \sum_{l} \sum_{j: l_{j}^{k} \subseteq J_{t_{i}^{k-N}}} 2^{k p} \int\left[M^{+} \chi_{I_{j}^{k}}\right]^{q} w \leq C \sum_{l} 2^{k p} \int\left[M^{+} \chi_{J_{l}^{k-N}}\right]^{q} w .
\end{aligned}
$$

To estimate IV we observe that

$$
\mathrm{IV} \leq C(\delta) 2^{k p} \sum_{i} w\left(I_{i}^{k-N}\right)+\left(\delta+C 2^{-N q}\right) 2^{k p} \sum_{i} \int\left[M^{+} \chi_{l_{i}^{k-N}}\right]^{q} w
$$




$$
\leq C 2^{k p} w\left(\Omega_{k-N}\right)+\frac{1}{2} S(k-N),
$$

choosing $\delta$ small and $N$ big enough. Combining III and IV we get

$$
S(k) \leq \frac{1}{2} S(k-N)+C 2^{k p} w\left(\Omega_{k-N}\right)+C 2^{k p} \sum_{l} \int\left[M^{+} \chi_{J_{l}^{k-N}}\right]^{q} w .
$$

Using Lemma 2

$$
\begin{aligned}
S_{M}=\sum_{k \leq M} S_{k} & \leq \frac{1}{2} S_{M}+C \int\left[T^{*+} f\right]^{p} w+C \int\left[M_{p, q}^{+}\left(M^{+} f\right)\right]^{p} w \\
& \leq \frac{1}{2} S_{M}+C\left(\int\left[T^{*+} f\right]^{p} w+\int\left[M^{+} f\right]^{p} w\right)
\end{aligned}
$$

and since $S_{M}<\infty$ (see Lemma 2), we get

$$
\int\left[M_{p, q}^{+}\left(T^{*+} f\right)\right]^{p} w \leq C\left(\int\left[T^{*+} f\right]^{p} w+\int\left[M^{+} f\right]^{p} w\right) .
$$

PROOF OF THEOREM 1. First we observe that $\left|T^{+} f\right| \leq T^{*+} f$, so it is enough to prove the theorem for $T^{*+}$. Let $f$ be a non-negative bounded function with compact support.

Let $\Omega_{k}=\left\{x: T^{*+} f(x)>2^{k}\right\}=\bigcup_{j} J_{j}^{k}$ where $J_{j}^{k}$, are the connected components of $\Omega_{k}$. Let us fix $(a, b)=J_{j}^{k}$. We partition $(a, b)$ as follows. Let $x_{0}=a$, and we choose $x_{i+1}$ such that $x_{i+1}-x_{i}=b-x_{i+1}$ and we let $I_{i}^{k}=\left(x_{i}, x_{i+1}\right)$. By 'the good lambda inequality' in [AFM, Lemma 2.7] we have that

$$
\left|E_{i}^{k}\right|=\left|\left\{x \in I_{i}^{k}: T^{*+} f(x)>2^{k+1}, M^{+} f(x) \leq \gamma 2^{k}\right\}\right| \leq C \gamma\left|I_{i}^{k}\right| \quad \text { for } 0<\gamma<1 .
$$

From $C_{q}^{+}$condition we have

$$
w\left(E_{i}^{k}\right) \leq C \gamma^{\epsilon} \int\left[M^{+} \chi_{I_{i}^{k} \cup I_{i+1}^{k}}\right]^{q} w
$$

Summing over all $i$ and using Lemma 1 we infer that

$$
\begin{aligned}
w(\{x & \left.\left.\in J_{j}^{k}: T^{*+} f(x)>2^{k+1}, M^{+} f(x) \leq \gamma 2^{k}\right\}\right) \\
& \leq C \gamma^{\epsilon} \sum_{i} \int\left[M^{+} \chi_{I_{i, j}^{k} \cup I_{i+1, j}^{k}}\right]^{q} w \leq C \gamma^{\epsilon} \int\left[M^{+} \chi_{J_{j}^{k}}\right]^{q} w .
\end{aligned}
$$

Now, summing over all $j$ we have that

$$
w\left(\left\{x \in \Omega_{k}: T^{*+} f(x)>2^{k+1}, M^{+} f(x) \leq \gamma 2^{k}\right\}\right) \leq C \gamma^{\epsilon} \sum_{j} \int\left[M^{+} \chi_{j_{j}^{k}}\right]^{q} w .
$$


Then by Lemma 3,

$$
\begin{aligned}
\int\left(T^{*+} f\right)^{p} w= & \sum_{k} \int_{\Omega_{k}-\Omega_{k+1}}\left(T^{*+} f\right)^{p} w \leq 2^{p} \sum_{k} 2^{k p} w\left(\Omega_{k}\right) \\
= & C \sum_{k} 2^{k p}\left[w\left(\left\{x \in \Omega_{k}: T^{*+} f>2^{k+1}, M^{+} f \leq \gamma 2^{k}\right\}\right)\right. \\
& \left.\quad+w\left(\left\{x \in \Omega_{k}: T^{*+} f>2^{k+1}, M^{+} f>\gamma 2^{k}\right\}\right)\right] \\
\leq & \sum_{j, k}\left(C \gamma^{\epsilon} 2^{k p} \int\left[M^{+} \chi_{J_{j}^{k}}\right]^{q} w\right) \\
& +C \sum_{k} 2^{k p} w\left(\left\{x \in \Omega_{k}: M^{+} f(x)>\gamma 2^{k}\right\}\right) \\
\leq & C \gamma^{\epsilon}\left[\int\left[T^{*+} f\right]^{p} w+\int\left[M^{+} f\right]^{p} w\right]+C \int\left[M^{+} f\right]^{p} w .
\end{aligned}
$$

Finally we prove that under the assumptions on $f$, we have that $\int\left[T^{*+} f\right]^{p} w<\infty$, and choosing $\gamma$ small enough we finish the proof. To see that $\int\left[T^{*+} f\right]^{p} w<\infty$, let supp $f \subset I=(a, b)$ and $I^{-}=(2 a-b, a)$. If $x<2 a-b$, then $T^{*+} f(x) \leq$ $C M^{+} f(x)$, so

$$
\int_{-\infty}^{2 a-b}\left[T^{*+} f\right]^{p} w \leq \int_{-\infty}^{2 a-b}\left[M^{+} f\right]^{p} w<\infty
$$

Since $T^{*+} f$ is a singular integral and $f$ is bounded, it is known that $\int_{I-\cup I} e^{\alpha T^{*+} f}<\infty$ for some $\alpha>0$. Thus

$$
\left|E_{\lambda}\right|=\left|\left\{x \in I^{-} \cup I: T^{*+} f(x)>\lambda\right\}\right| \leq C e^{-\lambda \alpha}\left|I^{-} \cup I\right|
$$

for all $\lambda>0$. Applying the $C_{q}^{+}$condition to the set $E_{\lambda}$ and the points $2 a-b, b$, $2 b-a$, we get

$$
w\left(E_{\lambda}\right) \leq C e^{-\lambda \alpha \epsilon} \int\left[M^{+} \chi_{I^{-} \cup / \cup I^{+}}\right]^{q} w,
$$

where $I^{+}=(b, 2 b-a)$. Integrating with respect to $\lambda$, using that $p<q$, and proceeding as in the final step of the proof of Lemma 1 , we have

$$
\int_{I-\cup I}\left[T^{*+} f\right]^{p} w \leq C \int\left[M^{+} \chi_{I-U I U I^{+}}\right]^{p} w<\infty .
$$

As observed in the introduction $A_{\infty}^{+} \subseteq \bigcap_{p>1} C_{p}^{+}$. We now show that the inclusion is proper. 
Proposition 1. Let $w \in A_{\infty}$, then $w \chi_{(-\infty, 0)} \in \bigcap_{p>1} C_{p}^{+}$.

PROOF. First we observe that $w \chi_{(-\infty, 0)} \notin A_{\infty}^{+}$. Let us consider $a<b<c$ such that $c-b<b-a$ and $E$ a mesurable set such that $E \subset(a, b)$. We have several cases

(i) $a<b<c<0$. In this case there is nothing to prove because

$$
A_{\infty} \Longrightarrow A_{\infty}^{+} \Longrightarrow \bigcap_{p>1} C_{p}^{+}
$$

(ii) $a<b<0<c$. There exist $\epsilon>0$ and $C>0$ such that

$$
\begin{aligned}
w \chi_{(-\infty, 0)}(E) & =w(E) \leq C\left(\frac{|E|}{b-a}\right)^{\epsilon} w(a, b) \leq C\left(\frac{|E|}{c-b}\right)^{\epsilon} \int_{a}^{b}\left[M^{+} \chi_{(a, b)}\right]^{p} w \\
& \leq C\left(\frac{|E|}{c-b}\right)^{\epsilon} \int_{-\infty}^{0}\left[M^{+} \chi_{(a, b)}\right]^{p} w \leq C\left(\frac{|E|}{c-b}\right)^{\epsilon} \int_{-\infty}^{0}\left[M^{+} \chi_{(a, c)}\right]^{p} w .
\end{aligned}
$$

(iii) $a<0<b<c$, and $b \leq-2 a$. Suppose that $E \subseteq(a, 0)$. Note that since $b-a \leq-3 a$,

$$
\begin{aligned}
w \chi_{(-\infty, 0)}(E) & =w(E) \leq C\left(\frac{|E|}{0-a}\right)^{\epsilon} w(a, 0) \leq C\left(\frac{|E|}{b-a}\right)^{\epsilon} \int_{a}^{0}\left[M^{+} \chi_{(a, 0)}\right]^{p} w \\
& \leq C\left(\frac{|E|}{c-b}\right)^{\epsilon} \int_{-\infty}^{0}\left[M^{+} \chi_{(a, c)}\right]^{p} w .
\end{aligned}
$$

If $E \nsubseteq(a, 0)$, then

$$
\begin{aligned}
w \chi_{(-\infty, 0)}(E) & =w(E \cap(-\infty, 0)) \leq C\left(\frac{|E \cap(-\infty, 0)|}{c-b}\right)^{\epsilon} \int_{-\infty}^{0}\left[M^{+} \chi_{(a, c)}\right]^{p} w \\
& \leq C\left(\frac{|E|}{c-b}\right)^{\epsilon} \int_{-\infty}^{0}\left[M^{+} \chi_{(a, c)}\right]^{p} w .
\end{aligned}
$$

(iv) $a<0<b<c$ and $b>-2 a$.

$$
w \chi_{(-\infty, 0)}(E) \leq w(E) \leq C\left(\frac{|E|}{b-a}\right)^{\epsilon} w(a, b) \leq C\left(\frac{|E|}{c-b}\right)^{\epsilon} w(a, b) .
$$

If we prove that $w(a, b) \leq C \int_{-\infty}^{0}\left[M^{+} \chi_{(a, c)}\right]^{p} w$, we have finished the proof. Using that $w$ satisfies the doubling condition and that $b>-2 a$ if and only if $a+b>b / 2$ we have

$$
\begin{aligned}
\int_{-\infty}^{0}\left[M^{+} \chi_{(a, c)}\right]^{p} w & \geq \int_{-\infty}^{0}\left[M^{+} \chi_{(a, b)}\right]^{p} w \geq \int_{-b}^{a}\left[M^{+} \chi_{(a, b)}\right]^{p} w=\int_{-b}^{a}\left(\frac{b-a}{b-x}\right)^{p} w \\
& \geq \int_{-b}^{a}\left(\frac{1}{2}\right)^{p} w \geq \frac{C}{2^{p}} w(-b, a) \geq C w(a, b) .
\end{aligned}
$$




\section{References}

[AFM] H. Aimar, L. Forzani and F. J. Martín Reyes, 'On weighted inequalities for one-sided singular integrals’, Proc. Amer. Math. Soc. 125 (1997), 2057-2064.

[FeSt] C. Fefferman and E. Stein, 'Some maximal inequalities', Amer. J. Math. 93 (1971), 107-115.

[Mu] B. Muckenhoupt, 'Norm inequalities relating the Hilbert transform to the Hardy-Littlewood maximal function', in: Functional analysis and approximation (Oberwolfach 1980), Internat. Ser. Number. Math. 60 (Birkhauser, Basel-Boston, Mass., 1981) pp. 219-231.

[MPT] F. J. Martín Reyes, L. Pick and A. de la Torre, 'A $A_{\infty}^{+}$condition', Can. J. Math 45 (1993), 1231-1244.

[S1] E. Sawyer, 'Weighted inequalities for the one-sided Hardy-Littlewood maximal function', Trans. Amer. Math. Soc. 297 (1986), 53-61.

[S2] _ _ 'Norm inequalities relating singular integrals and maximal functions', Studia Math. 75 (1983), 251-263.

FaMAF

Universidad Nacional de Córdoba (5000) Córdoba

Argentina

e-mail: sriveros@mate.uncor.edu
Análisis Matemático

Facultad de Ciencias

Universidad de Málaga

(29071) Málaga

Spain

e-mail: torre@anamat.cie.uma.es 